\title{
«Chacun connaît chacun»
}

\section{Françoise Verrey Bass}

Spécialiste retraitée en neurologie, membre FMH, présidente ASEM

Quelque part c'est presque un peu présomptueux de parler de congrès mondial; international serait plus juste. Aussi le terme d'écrivains manque de modestie, car nous sommes des médecins qui écrivent, aiment écrire, publient souvent, mais rares sont ceux qui deviennent célèbres. Et à ce moment-là ils sortent de l'association. Bref: je pense que cela suffirait d'intituler l'association: association internationale des médecins qui écrivent (autre chose que des articles médicaux). Mais le nom a été créé il y a 60 ans, en 1956, à Ascona. Notre rencontre a eu lieu du 14 au 18 septembre et nous étions tout contents que grâce aux liens renoués il y a un an, merci Sergio Marengo, le $60^{\mathrm{e}}$ anniversaire a pu être fêté dans un des pays fondateurs de l'époque, l'Italie. Les représentants italiens n'étaient plus venus à nos congrès depuis plusieurs années.

\section{Des rencontres internationales}

Nous eûmes aussi le plaisir de saluer le nouveau président bulgare et le président roumain. Nous retrouvâmes un cher ami polonais, qui revient fidèlement chaque année, où que nous soyons, traversant s'il le faut toute l'Europe en voiture pour nous retrouver au Portugal ou en Bretagne. Puis il y a aussi ce cher et fidèle ami d'Equateur, dont nous aimons beaucoup les écrits, heu, leur traduction... Malheureusement le Professeur du Mozambique ne revient plus, l'âge, la difficulté du voyage...

Nous sommes en général entre 40 et 50 personnes, ce qui fait que chacun connaît chacun, que nous devenons tous de vrais amis.

\section{Un congrès convivial avec des excursions culturelles}

Le congrès est devenu beaucoup plus simple et convivial qu'il y a encore une quinzaine d'années et nous pouvons saluer chaque fois quelques nouveaux et jeunes collègues, surtout de France ou d'Allemagne, mais cette fois aussi d'Italie. La quatrième grande délé- gation est venue comme d'habitude du Portugal. C'est un congrès, où les partenaires assistent aussi aux séances de lecture, et cette année encore, cela en valait la peine. Le sujet était: la médecine et l'art. Mais on peut parfaitement aussi lire quelque chose qui n'est pas en rapport avec le thème donné. Une partie du congrès est réservée à des excursions dans la région, ici une visite du musée de la soie de Lecco, ancienne fabrique de la famille Abegg de Suisse (cela vous rappelle-t-il quelque chose?), puis une grande virée sur le lac de Côme avec ensuite visite de la chapelle des

\section{Le troisième jour, dans le désordre, nous avons vu Bergame, juste après la fin d'une pluie torrentielle.}

cyclistes, où le Giro s'arrête obligatoirement chaque année pour une bénédiction, petit musée avec des objets qui ont appartenu aux grands du cyclisme, le vélo, une paire de gants etc. Le troisième jour, dans le désordre, nous avons vu Bergame, juste après la fin d'une pluie torrentielle. Nous avons pu faire la visite prévue de la ville sur son rocher et admirer l'architecture de transition d'une culture à l'autre, du sud au nord et de l'est à l'ouest. Puis nous avons eu une visite guidée de la très fameuse pinacothèque, l'Académie Carrara.

\section{L'année prochaine, ce sera la Bulgarie}

Le congrès commence le mercredi soir et se termine officiellement le samedi soir par une soirée un peu plus festive, agrémentée à Lecco par un concert de gospels. Si par le passé les grands repas étaient très importants, nous privilégions maintenant l'aspect culturel de la rencontre et surtout l'amitié!

Nous nous réjouissons beaucoup d'aller l'année prochaine pour la troisième fois en Bulgarie, cette fois-ci à Plovdiv, dans le sud de la Bulgarie, une des plus anciennes villes d'Europe, au cœur de la civilisation des Thraces, où se trouve par ailleurs un superbe théâtre romain. Le congrès aura lieu dans la deuxième moitié de septembre. Le thème prévu est: médecine et paix. 